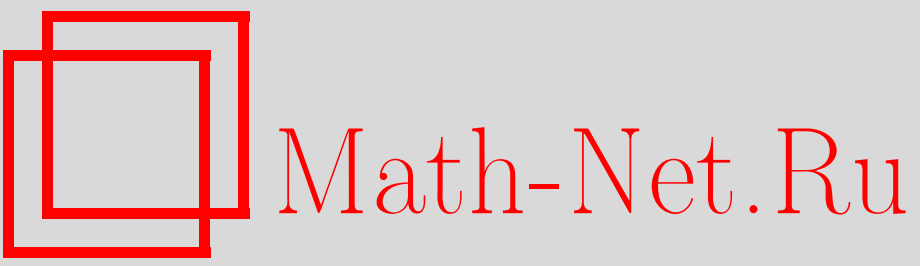

М. Л. Гервер, Е. А. Кудрявцева, Новое доказательство теорем об универсальной последовательности и экстремальных свойствах дискретных мер, УМH, 1997, том 52, выпуск 6, 153-154

DOI: https://doi.org/10.4213/rm898

Использование Общероссийского математического портала Math-Net.Ru подразумевает, что вы прочитали и согласны с пользовательским соглашением

http: //www . mathnet.ru/rus/agreement

Параметры загрузки:

IP : 18.234 .197 .8

26 апреля 2023 г., 13:06:25

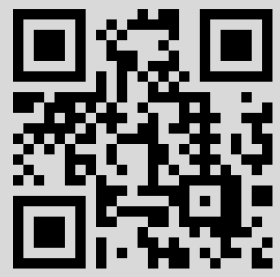




\title{
НОВОЕ ДОКАЗАТЕЛЬСТВО ТЕОРЕМ ОБ УНИВЕРСАЛЬНОЙ ПОСЛЕДОВАТЕЛЬНОСТИ И ЭКСТРЕМАЛЬНЫХ СВОЙСТВАХ ДИСКРЕТНЫХ МЕР
}

\author{
М. Л. ГЕРВЕР, Е. А. КУДРЯВЦЕВА
}

В статье предложен новый, опирающийся на принцип двойственности способ доказательства теорем из [1], [2].

Двойственные экстремальные задачи. На полуоси $s \geqslant 0$ рассмотрим неотрицательные меры Стилтьеса $d a=d a(s)$ с интегралом, равным 1 , и каждой такой мере сопоставим две функции:

$$
g(d a, t)=\int_{0}^{\infty} \frac{d a(s)}{s+t}, \quad t \geqslant 0, \quad h(d a, x)=\frac{2}{\pi} \int_{x}^{\infty} g(d a, t) d \sqrt{t}, \quad x \in[0,1] .
$$

Произвольно фиксируем положительную полунепрерьвную снизу функцию (мажсоранту) $M(t), t \in[0,1]$. Отнесем $d a$ к множеству $\mathscr{A}$, если $g(d a, t) \leqslant M(t)$ при всех $t \in[0,1]$. На $\mathscr{A}$ рассмотрим однопараметрическое (зависящее от параметра $x \in[0,1]$ ) семейство экстремальных задач:

$$
\text { найти } \sup h(d a, x) \text { по всем } d a \in \mathscr{A} \text {. }
$$

Физическую интерпретацию (1) можно найти в [1], [2].

Теперь рассмотрим пары $\left(b_{0}, d b=d b(t)\right)$, где $b_{0}$ - неотрицателњные числа, a $d b(t)$ - неотрицательные меры Стилтьеса на отрезке $[0,1]$, и каждой такой паре сопоставим функцию $r\left(b_{0}, d b, s\right)$, $s \geqslant 0$, и число $H\left(b_{0}, d b\right)$ :

$$
r\left(b_{0}, d b, s\right)=b_{0}+\int_{0}^{1} \frac{d b(t)}{s+t}, \quad H\left(b_{0}, d b\right)=b_{0}+\int_{0}^{1} M(t) d b(t) .
$$

На полуоси $s \geqslant 0$ фиксируем однопараметрическое (зависящее от параметра $x$ ) семейство функций (семейство минорант)

$$
I(s, x)=\frac{2}{\pi} \int_{x}^{\infty} \frac{d \sqrt{t}}{s+t}=\frac{2}{\pi} \frac{\operatorname{arctg} \sqrt{s / x}}{\sqrt{s}}, \quad x \in(0,1] ; \quad I(s, 0)=\frac{1}{\sqrt{s}} .
$$

Фиксируем $x>0$ в (1), положим $h(d a)=h(d a, x), I(s)=I(s, x), s \geqslant 0$, и отнесем к множеству $\mathscr{B}=\mathscr{B}(x)$ пары $\left(b_{0}, d b\right)$, для которых $r\left(b_{0}, d b, s\right) \geqslant I(s)$ при всех $s \geqslant 0$. Задача, двойственная к (1), формулируется так:

$$
\text { найти inf } H\left(b_{0}, d b\right) \text { по всем }\left(b_{0}, d b\right) \in \mathscr{B}(x) \text {. }
$$

ТЕОРема 1 (принцип двойственности). Верхняя и нижняя грани в (1), (2) достигаются и равны друг другу: $\max _{\mathscr{A}} h(d a, x)=\min _{\mathscr{B}(x)} H\left(b_{0}, d b\right), x \in(0,1]$.

ОПРЕДЕЛЕНИЕ. Особъми точками меры $d a \in \mathscr{A}$ назовем точки $t \in[0,1]$, в которых $g(d a, t)=M(t)$, а особыми точками пары $\left(b_{0}, d b\right) \in \mathscr{B}$ - точки $s \geqslant 0$, в которых $r\left(b_{0}, d b, s\right)=$ $I(s)$.

Работа выполнена при финансовой поддержке Российского фонда фундаментальных исследований (грант № 96-01-01852). 
КРИТЕРИЙ СОВПАДЕНИЯ $h(d a)$ и $H\left(b_{0}, d b\right)$. Для любой меры $d a \in \mathscr{A}$ u любой napы $\left(b_{0}, d b\right) \in \mathscr{B}$ выполняется неравенство $h(d a) \leqslant H\left(b_{0}, d b\right)$. Для совпадения $h(d a)$ и $H\left(b_{0}, d b\right)$ необходимо и достаточно, чтобы мера dа была сосредоточена в особых mочках $\left(b_{0}, d b\right)$, а мера $d b-\varepsilon$ особьх точках $d a$.

В последующих формулировках участвует универсальная (не зависящая от выбора мажоранты $M(t))$ убывающая и сходящаяся к 0 последовательность $X_{k} \in[0,1], k \geqslant 1$. Ее определение связано с полиномами Лежандра

$$
L_{0}(x)=1, \quad L_{1}(x)=x, \quad L_{n}(x)=\left(2-\frac{1}{n}\right) x L_{n-1}(x)-\left(1-\frac{1}{n}\right) L_{n-2}(x),
$$

а именно, $X_{k}-\kappa в$ адрат наименьшего положительного корня $L_{2 k+1}(x), k \geqslant 1$ :

$$
\begin{aligned}
& X_{1}=0.60000, X_{2}=0.28995, X_{3}=0.16471, X_{4}=0.10514, X_{5}=0.07265, \\
& X_{6}=0.05311, X_{7}=0.04048, X_{8}=0.03186, X_{9}=0.02572, X_{10}=0.02119, \ldots
\end{aligned}
$$

ОсНОвноЕ УТВеРЖДЕНИЕ ОБ ОСОБЫХ точках. Пусть $x \geqslant X_{k}$ uз (3), u nусть

$$
s_{j}, \quad 1 \leqslant j \leqslant m, \quad s_{1}>\cdots>s_{m} \geqslant 0
$$

являются особыми точками пары $\left(b_{0}, d b\right) \in \mathscr{B}=\mathscr{B}(x)$. Тогда $m \leqslant k$.

Из последнего неравенства, принципа двойственности и критерия совпадения $h(d a)$ и $H\left(b_{0}, d b\right)$ следует

Tеорема 2 (о дискретности $\left.d a^{\circ}\right)$. Пусть $x \geqslant X_{k}$ из (3), и пусть $\max _{\mathscr{A}} h(d a, x)$ достuгается на мере $d a^{\circ}$. Тогда da $^{\circ}$ сосредоточена в конечном числе точек $s_{1}^{\circ}>\cdots>s_{n}^{\circ} u$ $n=n(x) \leqslant k$.

Теорема 3 (о строгом максимуме). Для любой меры $d a \in \mathscr{A}$, не равной $d a^{\circ}$, выполняется строгое неравенство $h(d a)<h\left(d a^{\circ}\right)$.

Предположим дополнительно, что $\underline{\lim }_{t \rightarrow 0} t M(t)=0$. Тогда (см. теорему 2$) s_{n}^{\circ}>0$, и теоремы 2, 3 можно переформулировать следующим образом:

Для каждого $x \in(0,1]$ существуют и единственны такое $n=n(x)$ и такие

$$
a_{j}=a_{j}(x)>0, \quad 1 \leqslant j \leqslant n, \quad \sum a_{j}=1, \quad s_{1}=s_{1}(x)>\cdots>s_{n}=s_{n}(x)>0,
$$

что строгий максимум $h(d a, x)$ достигается на мере $d a=\sum_{1}^{n} a_{j} \delta\left(s-s_{j}\right) d s$, причем $n(x) \leqslant k$, ecлu $x \in\left[X_{k}, 1\right]$.

\section{СПИСОК ЛИТЕРАТУРЫ}

[1] Гервер М. Л., Кудрявцева Е. А. // Матем. сб. 1997. Т. 188. № 4. С. 3-56. [2] Гервер М. Л., Кудрявцева Е. А. // Докл. АН. 1997. Т. 356. № 1. С. 25-28.

Международный институт теории прогноза землетрясений и математической геофизики $\mathrm{PAH}$;

Принято редколлегией Московский государственньй университет им. М. В. Ломоносова 03.09.1997 E-mail: gerver@mitp.rssi.ru m.l@gerver.mccme.ru 